\title{
Erratum: Generalized quantum state discrimination problems [Phys. Rev. A 91, 052304 (2015)]
}

\author{
Kenji Nakahira ${ }^{\circledR}$, Kentaro Kato, and Tsuyoshi Sasaki Usuda
}

(Received 17 December 2020; published 5 January 2021)

DOI: 10.1103/PhysRevA.103.019902

The second paragraph below Eq. (18) of our work (which starts with "Slater's condition") should read: "Slater's condition is known to a sufficient condition under which, if the primal problem is convex, the optimal values of the primal and dual problems are the same [31]. Let $\lambda_{j}=1$ for each $j$; then, there exists $\hat{X}$ such that $\hat{X}-\hat{z}_{m}(\lambda)$ is positive definite for any $m \in \mathcal{I}_{M}$, and, thus, Slater's condition holds. Therefore, the optimal values of problems (2) and (12) are the same."

The first full paragraph below Eq. (27) (which starts with "We can see that") should read " $\left(\mu^{\star}, \Pi^{\star}\right)$ is called a saddle point, also referred to as a minimax solution, of $F(\mu, \Pi)$ if

$$
F\left(\mu^{\star}, \Pi\right) \leqslant F\left(\mu^{\star}, \Pi^{\star}\right) \leqslant F\left(\mu, \Pi^{\star}\right)
$$

holds for any $\mu \in \mathcal{P}$ and $\Pi \in \mathcal{M}^{\circ}$. A saddle point exists if and only if the so-called minimax theorem, that is,

$$
\max _{\Pi \in \mathcal{M}^{\circ}} \min _{\mu \in \mathcal{P}} F(\mu, \Pi)=\min _{\mu \in \mathcal{P}} \max _{\Pi \in \mathcal{M}^{\circ}} F(\mu, \Pi)
$$

holds, and this value equals $F\left(\mu^{\star}, \Pi^{\star}\right)$ [37]. We can see that if $\mathcal{M}^{\circ}$ is not empty, then the minimax theorem holds. Indeed, $\mathcal{M}^{\circ}$ and $\mathcal{P}$ are closed convex sets, and $F(\mu, \Pi)$ is a continuous convex function of $\mu$ for fixed $\Pi$ and a continuous concave function of $\Pi$ for fixed $\mu$, which are sufficient conditions for the minimax theorem to hold [37]. We call $\mu^{\star}$ and $\Pi^{\star}$, respectively, minimax probabilities and a minimax measurement."

[31] M. Slater, Cowles Comm. Discuss. Paper, Math. No. 403 (1950).
[37] I. Ekeland and R. Temam, Convex Analysis and Variational Problems (SIAM, North-Holland, 1999), Vol. 28. 